\title{
A Novel Strategy for P. falciparum Malaria based on Syk Kinase Inhibitors to Design Triple Artemisinin- based Combination Therapies (TACTs) to Counteract Delayed Parasite Clearance (Drug Resistance) Following Standard ACT Treatment
}

\author{
Francesco M. Turrini ${ }^{1 *}$, Philip S. Low ${ }^{2}$ and Huynh Dinh Chien ${ }^{3}$ \\ ${ }^{1}$ Department of Oncology, University of Turin, Turin, Italy \\ ${ }^{2}$ Department of Chemistry and Institute for Drug Discovery, Purdue University, West Lafayette IN 47907, USA \\ ${ }^{3}$ College of Health Science, Vin University, Hanoi, Vietnam
}

${ }^{\star}$ Corresponding author: Francesco M. Turrini, Department of Oncology, University of Turin Turin, Italy; Phone: +39 335 6591966; Email: francesco.turrini@unito.it Received: February 18, 2021; Accepted: February 25, 2021; Published: March 01, 2021

\section{Background}

Since their introduction in 2002, Artemisinin-based combination therapies (ACTs) represent the most effective antimalarial drugs available for the treatment of uncomplicated $P$. falciparum malaria. ACTs clearly contributed to the $60 \%$ decline of malaria mortality rates observed between 2000 and 2015, anyway, malaria eradication remains an elusive goal because from 2014 the decline of malaria incidence slowed dramatically, with some areas of the world now showing an increase in the incidence of malaria [1]. Although the precise causes of this trend are not easily definable, artemisinin resistance is considered a contributing factor $[2,3]$.

The major feature of ACTs relies on the combination of two drugs with different mechanisms of action and with markedly different pharmacokinetics: a fast acting/short lived artemisinin derivative combined with a slow acting/long lived standard antimalarial drug such as mefloquine, amodiaquine or piperaquine, commonly defined as "partner drugs".

The rationale of their antimalarial effectiveness is therefore based on the sequential action of two drugs: i) artemisinin derivatives acting within hours on most of parasite blood stages but relying on the formation of very short-lived radical intermediates (limited compliance); and ii) quinoline-based antimalarial drugs with a prolonged plasma half-life but affected by established forms of resistance. In addition, those partner drugs are prevalently active only on mature parasite stages. No synergistic interactions between the two components of ACTs have been observed, therefore ACTs rely on the sequential action of two different drugs.

Because the contribution of artemisinin to parasite clearance was dominant in the activities of the various ACTs, artesimisinin's remarkable potency is thought to have obscured the effects of mutations that would normally have rendered the parasite resistant to the partner drugs. While this in effect acted to suppress reemergence of resistance to piperaquine, mefloquine, and amodiaquine for many years, it also set up situation where the eventual advent of resistance to artemisinin would enable the rapid re-emergence of resistant mutations to the long-lived partner drugs. Indeed, resistance to ACTs is now being observed, and as no alternatives to ACTs are currently available, discovery of strategies for preserving and, possibly, improving the efficacy of the ACTs is increasingly urgent. For this reason, the efficacies of triple combination therapies (TACTs) involving artemisinin plus two former partner drugs are now being explored. [4].

\section{The Emergence of Artemisinin Resistance}

In 2009 the first evidence of artemisinin resistance was identified in a small cohort of patients in western Cambodia [5], where a delay in parasite clearance half-time was observed in patients treated with either artesunate monotherapy or artesunate plus mefloquine therapy. Increased recrudescence was observed only in the group treated with artesunate monotherapy, although, artemisinin as a single drug was always characterized by variable rates of treatment failure. It should be also considered that parasite clearance half-time has been demonstrated to be affected by different factors, including: the viability of the parasites detected by microscopy, the level of pre-existing antiplasmodial immunity and the stage of parasite maturation at time of treatment [6-9].

In 2014 a well-defined mutation in the PF3D7_1343700 kelch propeller domain ('K13-propeller') was shown to be strongly associated with delayed parasite clearance in vivo and with drug resistance in vitro [10].

Although complex mechanisms of resistance have been proposed [11], the causal relationships between K13-propeller mutations and artemisinin resistance are still unclear. Interestingly, in a large cohort 
Francesco M. Turrini (2021) A Novel Strategy for P. falciparum Malaria based on Syk Kinase Inhibitors to Design Triple Artemisinin-based Combination Therapies (TACTs) to Counteract Delayed Parasite Clearance (Drug Resistance) Following Standard ACT Treatment

study, low levels of antibodies directed to $P$. falciparum antigens correlated both with the prevalence of K13 mutations and with prolonged parasite clearance half time following ACT treatment [12] confirming a substantial role of host immunity on parasite clearance and, possibly, on the deselection of the K13 mutations. In agreement with this htypothesis, 3.4-fold higher pretreatment parasitemia in patients presenting both the K13 (C580Y) mutation and high prevalence of delayed parasite clearance following ACT (dihydropiperaquine-dihydroartemisinine) treatment [13].

Considering that the mechanism of resistance to artemisinin remains unknown and that no unequivocal marker for artemisinin resistance currently exists, the indicator to identify "suspected artemisinin partial resistance" to artemisinin is the proportion of patients who are parasitaemic on day 3 [14]. The discrimination between artemisinin resistance and/or resistance to the partner drug remains, therefore, unprecise.

\section{Clinical ACT Resistance}

ACTs are currently the treatment of choice for uncomplicated malaria, anyway, recent reports showed high rates of treatment failure leading the change of treatment policies in SMS countries [14-16] and in some African countries [17]. In general, ACT failure have been reported in areas where both artemisinin resistance and resistance to the partner drug has been shown to be endemic. In conclusion, besides the sub-clinical effects of artemisinin resistance, available ACTs are showing decreased clinical effectiveness in different countries where established resistance to the partner drug was documented. While several new drugs are undergoing clinical development, no replacement for current ACTs will be available for several years, raising serious concern regarding the ability to successfully treat all strains of malaria in the future.

\section{Triple Artemisinin Combination Therapies (TACTs)}

To counteract the loss of efficacy of ACT, an apparently promising solution to prevent further diffusion of ACT resistance lies in the development of new TACTs $[18,19]$.

In general, the design of TACTs has focused on either compensating for the insufficient activity of artemisinin adding a third fast-acting drug or compensating for the loss of activity of the slower acting partner drug by adding a related partner drug. Importantly, both strategies have been tested in clinical trials (Table 1).

In a test of the former approach, methylene blue, a fast-acting redox compound that is toxic to both intra-erythrocyte and gametocyte forms of $P$. falciparum, was shown to synergize with artesunate in vitro. While a subsequent clinical study in Burkina Faso of the triple combination of methylene blue plus artesunate plus amodiaquine showed fast parasite clearance, the therapy was compromised by some degree of hemolysis [20-22]. Consequently, this promising TACT may require further investigation to evaluate the risk of severe hemolysis in G6PD deficient individuals. In a recent example of the second approach, mefloquine, a commonly utilized partner drug in ACTs, was added to a dihydroartemisinin plus piperaquine ACT to augment the activity of the slower acting piperaquine [23]. Importantly, the triple combination therapy showed high efficacy in areas of Cambodia, Thailand and Vietnam known to have significant ACT resistance, and more importantly, the TACT was well tolerated. However, because both mefloquine and piperaquine resistant strains are diffuse in the same areas [14], in presence of delayd parasite clearance/artemisinin resistance, the selection of resistant strains to both partner drugs may occur.

Therefore, in our opinion, an ideal TACT, to prevent further resistance, should not only contain a fast-acting drug and a standard partner drug but also a totally novel third component with an orthogonal mechanism of action to which the parasite has never been exposed. Such a triple combination would also include the best properties of the existing ACTs plus the additional benefit of a new anti-malarial drug with a unique but potent mechanism of action.

\section{New TACTs based on Syk Inhibitors}

One possible TACT that could meet the above requirements would be comprised of dehydroartemisinin + piperaquine + an inhibitor of the erythrocyte tyrosine kinase, Syk, a red blood cell enzyme that plays a central role in the remodeling of the red cell membrane following $P$. falciparum invasion. From the early stages of parasite maturation, Syk phosphorylation of band 3 is markedly enhanced by the oxidative stress-induced inactivation of the erythrocyte's tyrosine phosphatases and oxidative cross-linking of band 3 [24-26]. This elevated tyrosine phosphorylation of band 3 is then known to cause radical destabilization of the membrane, which in turn has been demonstrated to be essential for the effective egress of the merozoites and for the propagation of the infection to new erythrocytes [27]. Thus, treatment of $P$. falciparum-infected erythrocytes with a Syk kinase inhibitor has been demonstrated to prevent parasite egress from the red blood cell, thereby terminating the parasite's life cycle.

On the other hand, Syk inhibitors act synergistically in combination with various artemisinin derivatives through the accumulation of redox active hemichromes [28]. Therefore, Syk inhibitors exert at least two relevant effects on infected erythrocytes: they quickly catalyze the activation of artemisinin in its pharmaceutically active radical form and, lately, they inhibit the parasite egress phase.

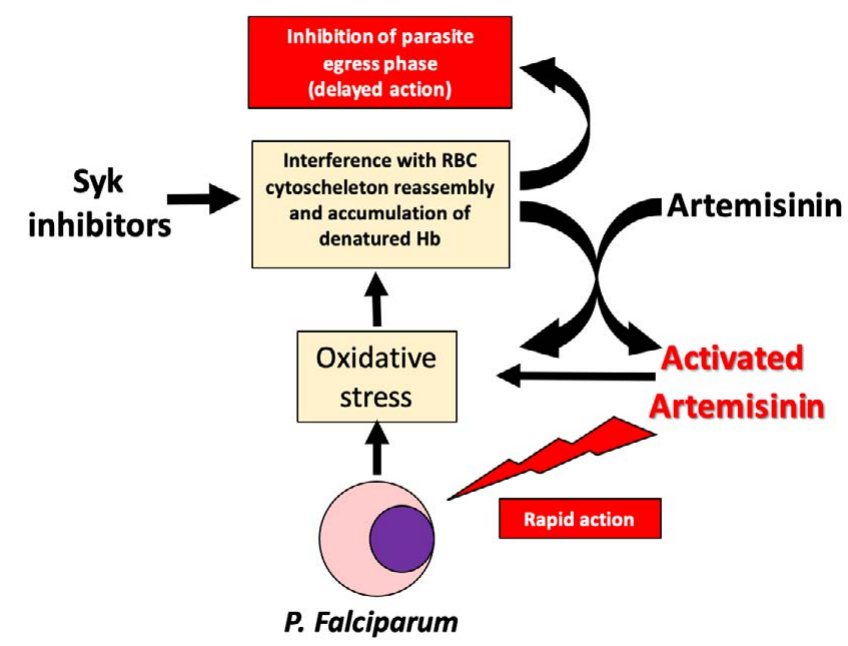

Figure 1: Scheme showing the complex mechanism of action of Syk inhibitors and their interactions with ACTs 
Francesco M. Turrini (2021) A Novel Strategy for P. falciparum Malaria based on Syk Kinase Inhibitors to Design Triple Artemisinin-based Combination Therapies (TACTs) to Counteract Delayed Parasite Clearance (Drug Resistance) Following Standard ACT Treatment

Table 1: Different strategies for TACTs development.

\begin{tabular}{|c|l|l|}
\hline \multicolumn{1}{|c|}{ ADDED DRUG } & \multicolumn{1}{|c|}{ EXPECTED EFFECTS } & COMMENTS \\
\hline $\begin{array}{c}\text { 1) Short-lived,freeradical drugsacting in parallel } \\
\text { with artemisinin (methyleneblue) }\end{array}$ & $\begin{array}{l}\text { Enhancement of the effectivenessoftheACTwith thepotentialto } \\
\text { compensate forartemisininresistance }\end{array}$ & $\begin{array}{l}\text { Free radical drugs can be hemolytic in G6PD deficient subjects. } \\
\text { Furtherstudiesmaybe needed to evaluatetherisk }\end{array}$ \\
\hline $\begin{array}{c}\text { 2) Long-lived drugs currently utilized as partner } \\
\text { drugs (mefloquine, amodiaquine, etc) }\end{array}$ & $\begin{array}{l}\text { Enhancement of the effectivenessoftheACT } \\
\text { compensating for the resistancetotheoriginal partner drug }\end{array}$ & $\begin{array}{l}\text { Establishedresistancetoall availablepartnerdrugs.Riskof } \\
\text { selecting multi-resistant parasites (?) }\end{array}$ \\
\hline $\begin{array}{c}\text { 3) Targetingahostenzyme topreventtheselection } \\
\text { of resistant parasites (Sykinhibitors) }\end{array}$ & $\begin{array}{l}\text { Fast synergistic action on artemisinin and delyed effect on the egress } \\
\text { phase to potentiatetheefficacy ofboth components of ACTs. [26] }\end{array}$ & $\begin{array}{l}\text { Needoffurtherclinicaldatato determine the clinical efficacy in areas } \\
\text { with demonstrated ACT resistance. }\end{array}$ \\
\hline
\end{tabular}

Our proposal for addition of a Syk kinase inhibitor as the third component of a TACT would successfully contribute to a totally orthogonal mechanism of action that should minimize the emergence of resistant strains to any of the TACT components (Figure 1).

A clinical trial to assess safety and effectiveness of a TACT composed by dihydroartemisinin, piperaquine and imatinib (a drug with a relevant inhibitory activity on Syk) is currently undergoing in Vietnam (in a site with demonstrated delayed parasite clearance) and Laos (no delayed parasite clearance). Good safety and effectiveness have been observed in a relatively small cohort of patients, the results will be published in short.

\section{Concluding Remarks}

- ACT resistance is a tangible worldwide menace.

- ACT effectiveness is based on the result of the sequential action of two different drugs. Any factor (artemisinin resistance and/ or partner drug resistance, changes of the immune status of patients, etc.) capable to interfere with this mechanism of action may determine treatment failure.

- New strategies to counterbalance the demonstrated default of both components of ACTs should be rapidly identified and tested.

- TACTs have been introduced to counteract ACT resistance but the development of multiresistant parasites should be considered very carefully.

- Human Syk inhibitors, acting on a non-parasite target and potentiating the action of artemisinin should not lead to the selection of resistant strains.

\section{References}

1. World malaria report 2019.

2. Ashley EA, Dhorda M, Fairhurst RM, Amaratunga C, Lim P, et al. (2014) Spread of artemisinin resistance in Plasmodium falciparum malaria. N Engl J Med 371: 411-23. [crossref]

3. Imwong M, Suwannasin K, Kunasol C, Sutawong K, Mayxay M, et al. (2017) The spread of artemisinin-resistant Plasmodium falciparum in the Greater Mekong subregion: a molecular epidemiology observational study. Lancet Infect Dis 17: 4917. [crossref]

4. van der Pluijm RW, Tripura R, Hoglund RM, Aung Pyae Phyo, Dysoley Lek, et al. (2020) Triple artemisinin-based combination therapies vs artemisinin- based combination therapies for uncomplicated Plasmodium falciparum malaria: A multicentre, open-label, randomized clinical trial. The Lancet 395(10233): 1345-1360 [crossref]

5. Dondorp AM, Nosten F, Yi P, Das D, Phyo AP, et al. (2009) Artemisinin Resistance in Plasmodium falciparum Malaria. N Engl J Med 361: 455-467. [crossref]

6. Khoury D, Zaloumis S, Grigg M, Haque A, Davenport M. (2020) Malaria Parasite
Clearance: What Are We Really Measuring? Trend in Parasitology. Volume 36 (5): 413-426. [crossref]

7. Nicholas J. White, (2017) Malaria parasite clearance. Malaria Journal 16: 88.

8. White NJ. (2011) Determinants of relapse periodicity in Plasmodium vivax malaria. Malar J 11; 10: 297. [crossref]

9. Hastings, I.M., Hodel, E.M. (2014) Pharmacological considerations in the design of anti-malarial drug combination therapies - is matching half-lives enough? Malar J 13: 62. [crossref]

10. Ariey F, Witkowski B, Amaratunga C, Beghain J, Langlois AC, et al. (2014) Jan A molecular marker of artemisininresistant Plasmodium falciparum malaria. Nature 2;505(7481): 50-5. [crossref]

11. Mbengue A, Bhattacharjee S, Pandharkar T, Liu H, Estiu G, et al. (2015) A molecualar mechanism of artemisinin resistance in Plasmodium falciparum malaria. Nature 520(7549): 683-687 [crossref]

12. Ataíde R, Powell R, Moore K, McLean A, Phyo AP, et al. (2017) Declining Transmission and Immunity to Malaria and Emerging Artemisinin Resistance in Thailand: A Longitudinal Study. J Infect Dis 216(6): 723-731. [crossref]

13. Pau MC, Pantaleo A, Tsamesidis I, et al. (2019) Clinical impact of the two ART resistance markers, K13 gene mutations and DPC3 in Vietnam. PLoS One 14(4): e0214667. [crossref]

14. WHO report - https: //www.who.int/docs/defaultsource/ documents/publications/ gmp/who-cds-gmp-2018-26-eng.pdf?ua=1

15. van der Pluijm, R. W., Imwong, M., Chau, N. H., Hoa, N. T., Thuy-Nhien, N. T., et al (2019) Determinants of dihydroartemisinin-piperaquine treatment failure in Plasmodium falciparum malaria in Cambodia, Thailand, and Vietnam: a prospective clinical, pharmacological, and genetic study. The Lancet. Infectious diseases, 19(9), 952-961. [crossref]

16. Saunders DL, Vanachayangkul P, Lon C. (2014) Dihydroartemisinin-Piperaquine Failure in Cambodia. N Engl J Med 371: 484-485 [crossref]

17. Nsanzabana. (2019) Resistance to Artemisinin Combination Therapies (ACTs): Do Not Forget the Partner Drug! Trop. Med Infect Dis 4: 26; [crossref]

18. White NJ. (2019) Triple artemisinin-containing combination anti-malarial treatments should be implemented now to delay the emergence of resistance. Malar J 18(1): 338 .

19. Rosenthal JP, Are three drugs for malaria better than two? The Lancet 395(10233), 1316-1317.

20. Zoungrana A, Coulibaly B, Sié A, Walter-Sack I, Mockenhaupt FP, et al. (2008) Safety and efficacy of methylene blue combined with artesunate or amodiaquine for uncomplicated falciparum malaria: a randomized controlled trial from Burkina Faso. PLoS One 3(2). [crossref]

21. Coulibaly B, Pritsch M, Bountogo M, Meissner PE, Nebié E, et al. (2015) Efficacy and safety of triple combination therapy with artesunate-amodiaquinemethylene blue for falciparum malaria in children: a randomized controlled trial in Burkina Faso. J Infect Dis 211(5): 689-97.

22. Müller O, Lu G, Jahn A \& Mockenhaupt FP. (2019) How worthwhile is methylene blue as a treatment of malaria? Expert Review of Anti-infective Therapy 17(7): 471-473.

23. van der Pluijm RW et al. (2020) Triple artemisinin-based combination therapies versus artemisinin-based combination therapies for uncomplicated Plasmodium falciparum malaria: a multicentre, open-label, randomised clinical trial. Lancet 395: 1345-60. [crossref]

24. Bordin B, Ion-Popa F, Brunati AM, Clari G, Low PS. (2005) Effector-induced Sykmediated phosphorylation in human erythrocytes. Biochimica et Biophysica Acta 1745: $20-28$ 
Francesco M. Turrini (2021) A Novel Strategy for P. falciparum Malaria based on Syk Kinase Inhibitors to Design Triple Artemisinin-based Combination Therapies (TACTs) to Counteract Delayed Parasite Clearance (Drug Resistance) Following Standard ACT Treatment

25. Pantaleo A, Ferru E, Pau MC, Khadjavi A, Mandili G, et al. (2016) Band 3 Erythrocyte Membrane Protein Acts as Redox Stress Sensor Leading to Its Phosphorylation by $\mathrm{p}$ (72) Syk. Oxid Med Cell Longev 6051093. [crossref]

26. Pantaleo A, Ferru E, Giribaldi G, Mannu F, Carta F, et al. (2009) Oxidized and poorly glycosylated band 3 is selectively phosphorylated by Syk kinase to form large membrane clusters in normal and G6PD-deficient red blood cells. Biochem J 1;418(2): 359-67.
27. Pantaleo A, Kesely KR, Pau MC, Tsamesidis I, Schwarzer E, (2017) Syk inhibitors interfere with erythrocyte membrane modification during $P$ falciparum growth and suppress parasite egress. Blood 130(8): 1031-1040. [crossref]

28. Tsamesidis I, Reybier K, Marchetti G, et al. (2020) Syk Kinase Inhibitors Synergize with Artemisinins by Enhancing Oxidative Stress in Plasmodium falciparumParasitized Erythrocytes. Antioxidants 9(8): 753. [crossref]

\section{Citation:}

Turrini FM, Low PS, Chien HD (2021) A Novel Strategy for P. falciparum Malaria based on Syk Kinase Inhibitors to Design Triple Artemisinin-based Combination Therapies (TACTs) to Counteract Delayed Parasite Clearance (Drug Resistance) Following Standard ACT Treatment. Interventional Medicine and Clinical Imaging Volume 4(1): 1-4 